\title{
Global metabolomic profiling of uterine leiomyomas
}

\author{
Hanna-Riikka Heinonen ${ }^{1}$, Miika Mehine ${ }^{1}$, Netta Mäkinen${ }^{1}$, Annukka Pasanen ${ }^{2}$, Esa Pitkänen ${ }^{1}$, Auli Karhu ${ }^{1}$, \\ Nanna S Sarvilinna ${ }^{3}$, Jari Sjöberg ${ }^{3}$, Oskari Heikinheimo ${ }^{3}$, Ralf Bützow ${ }^{2}$, Lauri A Aaltonen ${ }^{\star 1}$ and Eevi Kaasinen ${ }^{\star} 4$ \\ ${ }^{1}$ Department of Medical and Clinical Genetics and Genome-Scale Biology Research Program, University of Helsinki, P.O. Box 63, \\ Helsinki FIN-00014, Finland; ${ }^{2}$ Department of Pathology, University of Helsinki and Helsinki University Hospital, P.O. Box 21, \\ Helsinki FIN-00014, Finland; ${ }^{3}$ Department of Obstetrics and Gynaecology, University of Helsinki and Helsinki University Hospital, \\ P.O. Box 140, Helsinki FIN-00029, Finland and ${ }^{4}$ Division of Functional Genomics, Department of Medical Biochemistry and \\ Biophysics, Karolinska Institutet, Scheeles Väg 2, Stockholm SE-17177, Sweden
}

Background: Uterine leiomyomas can be classified into molecularly distinct subtypes according to their genetic triggers: MED12 mutations, HMGA2 upregulation, or inactivation of $\mathrm{FH}$. The aim of this study was to identify metabolites and metabolic pathways that are dysregulated in different subtypes of leiomyomas.

Methods: We performed global metabolomic profiling of 25 uterine leiomyomas and 17 corresponding myometrium specimens using liquid chromatography-tandem mass spectroscopy.

Results: A total of 641 metabolites were detected. All leiomyomas displayed reduced homocarnosine and haeme metabolite levels. We identified a clearly distinct metabolomic profile for leiomyomas of the $\mathrm{FH}$ subtype, characterised by metabolic alterations in the tricarboxylic acid cycle and pentose phosphate pathways, and increased levels of multiple lipids and amino acids. Several metabolites were uniquely elevated in leiomyomas of the FH subtype, including N6-succinyladenosine and argininosuccinate, serving as potential biomarkers for FH deficiency. In contrast, leiomyomas of the MED12 subtype displayed reduced levels of vitamin $\mathrm{A}$, multiple membrane lipids and amino acids, and dysregulation of vitamin $\mathrm{C}$ metabolism, a finding which was also compatible with gene expression data.

Conclusions: The study reveals the metabolomic heterogeneity of leiomyomas and provides the requisite framework for strategies designed to target metabolic alterations promoting the growth of these prevalent tumours.

Uterine leiomyomas are benign, yet extremely common tumours affecting up to $70 \%$ of women by age 50 years (Baird et al, 2003). Although the majority are asymptomatic, approximately $25 \%$ of affected women display symptoms including abnormal uterine bleeding and abdominal pain that require medical attention (Stovall, 2001). As conservative therapies leave room for improvement, patients frequently end up undergoing hysterectomy. Consequently, the economic and health impact of leiomyomas is enormous: the estimated annual health-care-related costs of uterine leiomyomas range from 6 to 34 billion USD in the United States (Cardozo et al, 2012).
Leiomyomas are oestrogen- and progesterone-dependent tumours that consist of slowly proliferating smooth muscle cells surrounded by abundant extracellular matrix (Malik et al, 2010). An affected uterus may contain multiple leiomyomas that vary greatly in size and may exceed $20 \mathrm{~cm}$ in diameter (Cramer and Patel, 1990). Molecular analyses have revealed that leiomyomas harbour mutually exclusive driver mutations (Markowski et al, 2012; Mehine et al, 2014; Kämpjärvi et al, 2016). The most frequent of these are mediator complex subunit 12 (MED12) mutations (Mäkinen et al, 2011) and high mobility group AT-hook 2 (HMGA2) translocations (Schoenmakers et al, 1995), which

*Correspondence: Dr LA Aaltonen; E-mail: lauri.aaltonen@helsinki.fi or Dr E Kaasinen; E-mail: eevi.kaasinen@ki.se

Received 7 June 2017; revised 18 September 2017; accepted 21 September 2017; published online 26 October 2017

(C) 2017 Cancer Research UK. All rights reserved 0007 - 0920/17 
affect approximately $80 \%$ and $10 \%$ of uterine leiomyomas, respectively (Mehine et al, 2014). Heterozygous germline mutations in the tricarboxylic acid (TCA) cycle enzyme fumarate hydratase $(F H)$ cause hereditary leiomyomatosis and renal cell cancer (HLRCC) syndrome, characterised by predisposition to type 2 papillary renal cell carcinoma, as well as uterine and cutaneous leiomyomas (Tomlinson et al, 2002). These syndromic tumours display a somatic second-hit mutation in $F H$, resulting in the complete loss of $\mathrm{FH}$ activity in neoplastic cells. Biallelic inactivation of $\mathrm{FH}$ accounts for only $1 \%$ of sporadic leiomyomas (Lehtonen et al, 2004). Different downstream mechanisms are likely to lead to the development of leiomyomas, as these three genetic-driver changes result in clearly distinct gene expression profiles (Mehine et al, 2016).

Reprogramming of metabolic pathways is a hallmark feature of neoplastic growth and therapeutic targeting of metabolic alterations is a promising strategy to control the process (Tennant et al, 2010; Pavlova and Thompson, 2016). To our knowledge, no prior studies exploring the whole metabolome of leiomyomas exist. Here we used untargeted metabolomic analysis to determine the pathophysiology of leiomyomas and the metabolic processes that enable tumour growth and development. This study explored the global metabolomic profile of 25 leiomyomas, harbouring different genetic-driver alterations including defects in $F H, M E D 12$, or HMGA2, and 17 respective normal myometrium samples. Furthermore, we integrated metabolomic and gene expression data to gain a deeper understanding of the molecular networks that are dysregulated in leiomyomas. We identified several metabolic alterations common for all leiomyomas and distinctive characteristics for leiomyomas of the $F H$ and MED12 subtypes.

\section{MATERIALS AND METHODS}

Subjects. The study material was derived from sample collections described in our previous work (Mäkinen et al, 2011; Heinonen et al, 2014; 2017; Mehine et al, 2016). Briefly, uterine leiomyoma and corresponding myometrial tissue samples were collected from patients undergoing hysterectomy in Finland between 2002 and 2015 and stored fresh frozen at $-80^{\circ} \mathrm{C}$. Samples from five patients (series M) were obtained anonymously with the approval of the director of the health-care unit. The other patients (series B, My, and $\mathrm{N}$ ) signed an informed consent, and clinical data were available from their patient records. The appropriate Ethics Review Board of Helsinki University Hospital, Finland approved this study.

Sample selection. The study samples were selected based on our previous work: seven leiomyomas were $F H$ deficient (three from HLRCC patients), seven harboured a mutation in MED12, two displayed HMGA2 overexpression, and nine were mutation negative for all three, henceforth termed triple wild-type leiomyomas. These 25 leiomyomas were obtained from 17 patients from whom normal myometrium samples were also included in the study.

All samples were screened for MED12 hotspot mutations using Sanger sequencing (Kämpjärvi et al, 2016). Deletions and rearrangements affecting $F H$ or HMGA2 loci were detected using whole-genome sequencing and/or SNP arrays (Supplementary Table S1) (Mehine et al, 2016). All tumours with 1q deletion and corresponding normal samples were screened for $F H$ mutations using Sanger sequencing (Supplementary Table S1) (Kämpjärvi et al, 2016). The relative quantification of HMGA2 expression was determined in all samples with rearrangements or deletions affecting HMGA2 using gene expression data (Mehine et al, 2016) or HMGA2 qPCR, performed on a 7500 Fast Real-Time PCR System with Assay No. Hs04397751_m1 (Applied Biosystems,
Darmstadt, Germany), using 18S rRNA as endogenous control (Supplementary Table S1).

Gene expression profiling. A total of 94 leiomyomas, including 10 with biallelic loss of $F H, 34$ with a MED12 mutation, 27 with an HMGA2 rearrangement and 23 triple wild-type leiomyomas, and 60 corresponding myometrium samples were assayed with Affymetrix GeneChip Human Exon 1.0 ST arrays. The gene expression profiling is described in detail in Mehine et al (2016).

Metabolomic profiling. Metabolomic profiling was performed by Metabolon Inc. (Durham, NC, USA) as described previously (Mesnage et al, 2016). Briefly, samples were analysed using four independent platforms: two reverse phase/ultra-performance liquid chromatography-tandem mass spectroscopy (RP/UPLC-MS/MS) methods with positive ion mode electrospray ionisation (ESI) chromatographically optimised separately for hydrophilic and hydrophobic compounds, one for analysis by RP/UPLC-MS/MS with negative ion mode ESI, and one for analysis by hydrophilic interaction chromatography/UPLC-MS/MS with negative ion mode ESI. All methods used an ACQUITY UPLC (Waters, Milford, MA, USA) and a Q-Exactive high-resolution/accurate mass spectrometer (Thermo Scientific Inc., Waltham, MA, USA) interfaced with a heated electrospray ionisation (HESI-II) source and Orbitrap mass analyser operated at 35000 mass resolution. A pooled matrix sample of each experimental sample served as a technical replicate; extracted water samples served as process blanks; and a cocktail of quality-control standards spiked into every analysed sample allowed instrument performance monitoring and aided chromatographic alignment. Compounds were identified by comparison to library entries of purified standards or recurrent unknown entities. Peaks were quantified using area under the curve.

To replicate the clustering results, a targeted analysis of 102 metabolites on 10 normal myometrium samples of uterine leiomyoma patients was carried out at the Metabolomics Unit, Technology Centre, Institute for Molecular Medicine Finland FIMM, University of Helsinki as previously described (Nikkanen et al, 2016).

Statistical analysis. Statistical analyses were performed with Partek Genomics Suite version 6.6 (Partek Inc., St Louis, MO, USA). Raw data of each metabolite were rescaled to set the median to 1 and $\log _{2}$ transformed. Missing values were imputed with the minimum observed value for each compound. Principal component analysis (PCA) was performed for all samples. Unsupervised hierarchical clustering analysis (cosine dissimilarity) was performed using all metabolites. The PCA and the unsupervised hierarchical clustering analysis revealed five outlier samples (M4 $\mathrm{m} 2, \mathrm{M} 4 \mathrm{~m} 3, \mathrm{M} 4 \mathrm{~N}, \mathrm{M} 11 \mathrm{~m} 2$, and M11 N), all of which were derived from two individual patients (M4 and M11). Both normal samples were also outliers in the replication data set (Supplementary Figure S1). These outlier samples were interpreted as biological outliers and therefore included in the subsequent analyses.

To evaluate whether tumour size (largest diameter) correlated with any of the metabolite levels, we calculated Pearson's correlation coefficients. A two-way ANOVA, with tissue type and patient-of-origin as factors, was constructed to identify metabolites that were dysregulated between leiomyomas and the corresponding myometrium samples. A one-way ANOVA, with tissue type as a factor, was constructed to identify metabolites that were dysregulated in each leiomyoma subtype (FH, MED12, $H M G A 2$, and triple wild type) as compared with all myometrium samples. The 20 most significant metabolites from each comparison, yielding 63 unique metabolites, were used for supervised hierarchical clustering analysis (cosine dissimilarity) of all tumour and normal samples. To identify the most uniquely dysregulated 
metabolites for leiomyomas of MED12 and FH subtypes, two three-way ANOVA tests, with mutation status (mutation positive or negative), tissue type, and patient-of-origin as factors, were constructed that compared each subtype against the rest of the leiomyoma and myometrium samples. To identify dysregulated main categories of metabolites between each leiomyoma subtype (FH, MED12, HMGA2, and triple wild type) and all myometrium samples, we used the Partek GO-ANOVA function (metabolite set ANOVA) with metabolites grouped into nine main categories. To explore whether any of the 86 metabolic pathways were significantly dysregulated, a pathway enrichment analysis was performed with the significant metabolites $(q$-value $<0.1)$ for each leiomyoma subtype. False discovery rate control was used to correct for multiple testing (Benjamini and Hochberg, 1995).

Ingenuity pathway analysis. Ingenuity pathway analysis (IPA) was carried out with each set of significantly dysregulated metabolites $(q$-value $<0.1)$ and each set of significantly differentially expressed genes $(q$-value $<0.1$, $\mid$ fold change $(\mathrm{FC}) \mid>1.5)$ using IPA software version 33559992 (Qiagen, Redwood City, CA, USA). We used IPA's comparison tool to identify pathways that were significantly dysregulated in both the metabolomic and gene expression data ( $P$-value $<0.05$ in both) for leiomyomas of the $F H$, $M E D 12$, and HMGA2 subtypes. We excluded the triple wild-type leiomyomas from the analyses due to their heterogeneity in the clustering analyses. Ingenuity Knowledge Base (Genes + Endogenous Chemicals) was used as a reference set.

\section{RESULTS}

LC-MS/MS identified 588 named and 53 unnamed metabolites among the 25 leiomyoma and 17 myometrium samples (Figure 1).
Genetic-driver changes had stronger effect on global metabolomic profiles than menopause status or hormone treatment of the patients (Supplementary Figure S2A). Leiomyomas of the FH subtype displayed a clearly distinct metabolomic profile according to the PCA (Figure 1) and the hierarchical clustering analysis (Supplementary Figure S2A). Leiomyomas of the FH, MED12, and HMGA2 subtypes were distinguishable from each other and from the myometrium based on abundancies of metabolites belonging to multiple different categories (Figure 2). The metabolites that most significantly correlated with tumour size were tryptophan betaine $(r=0.65,95 \%$ confidence interval 0.35 to $0.83, q$-value $=0.082)$ and glucose $(r=-0.64,95 \%$ confidence interval -0.83 to -0.33 , $q$-value $=0.089$, Supplementary Table S2).

To identify dysregulated metabolites in the complete set of leiomyomas, we compared all 25 leiomyomas against the corresponding 17 myometrial tissue specimens. Although patient-oforigin was a major source of variation across all metabolites (Supplementary Figure S2B), 70 metabolites were significantly dysregulated between leiomyoma and myometrium samples across all patients ( $q$-value $<0.1$, Supplementary Table S3). The most significant metabolite was homocarnosine (FC - 14.7), downregulated in all leiomyomas regardless of subtype (Figure 3). To identify dysregulated metabolites in leiomyomas of the FH, MED12, $H M G A 2$, and triple wild-type subtypes, we compared leiomyomas of each subtype against all myometrial tissue specimens. This revealed $170,104,35$, and 22 significant metabolites, respectively ( $q$-value $<0.1$, Supplementary Table S3). Most of the significantly dysregulated metabolites were increased in leiomyomas of the $F H(118 / 170$; $69 \%)$ and HMGA2 $(21 / 35 ; 60 \%)$ subtypes, whereas in the MED12 $(15 / 104 ; 14 \%)$ and triple wild-type subtypes $(4 / 22,18 \%)$ only a small proportion of the significantly aberrant metabolite levels showed increase (Figures 1C and D and 2). Owing to the limited number of HMGA2-overexpressing leiomyomas, we describe only changes
A

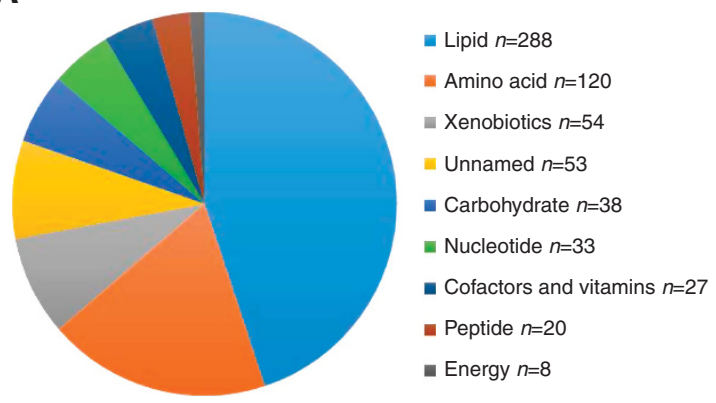

C

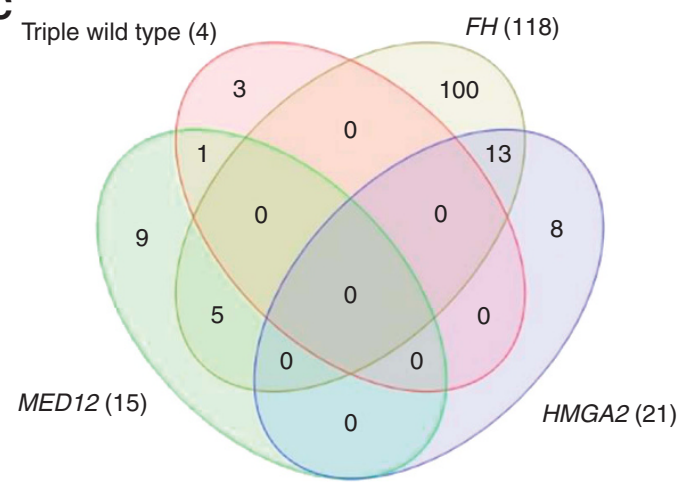

B

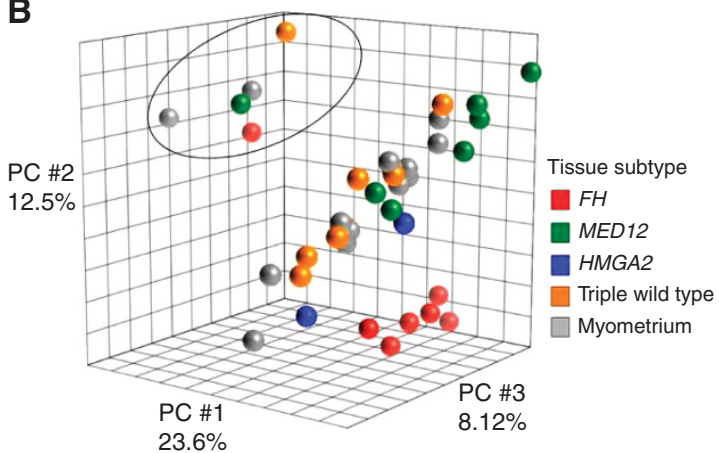

D Triple wild type (18) $F H(52)$

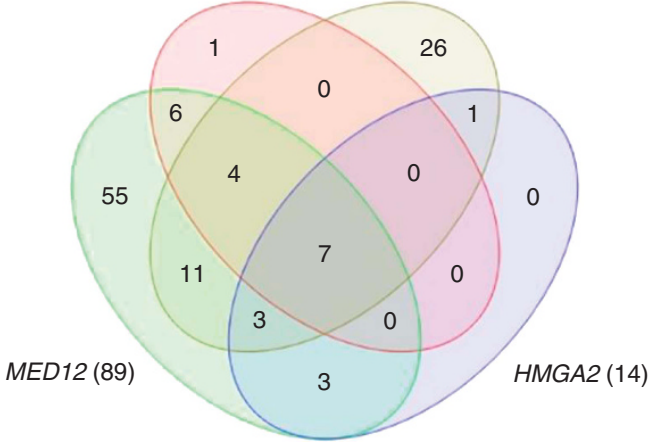

Figure 1. Overview of the metabolomic data. (A) A total of 641 metabolites were identified in the study. (B) Principal component analysis revealed five outlier samples (circle) and grouping of leiomyomas of the $\mathrm{FH}$ subtype together, while the rest of the samples were intermixed. Venn diagrams illustrating the number of significantly (C) increased and (D) decreased metabolites (q-value <0.1) among leiomyomas of the FH, MED12, HMGA2, and triple wild-type subtypes. 

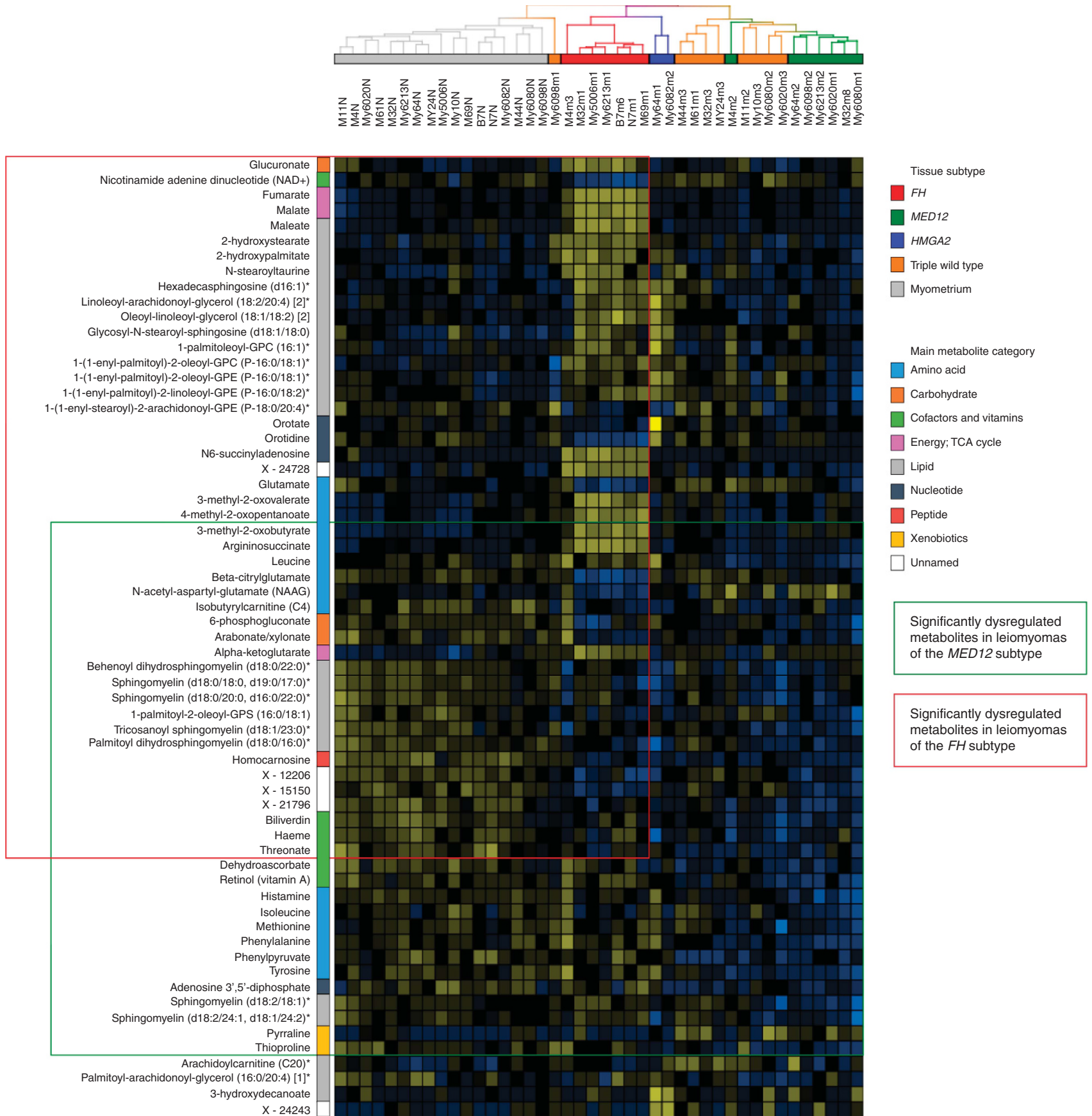

Figure 2. Supervised hierarchical clustering analysis with 63 metabolites comprising the 20 most significant metabolites of each leiomyoma subtype.

supported by both metabolic and expression data in this subtype or detectable in multiple subtypes.

Many cofactors and vitamins were altered in all leiomyomas or in specific subtypes. Haeme and biliverdin, which link to haemoglobin and porphyrin metabolism, were reduced in all leiomyoma subtypes (Supplementary Figure S3, Supplementary Table S3). Ascorbate (vitamin C) and aldarate metabolism was altered in leiomyomas of the MED12 and triple wild-type subtypes (Table 1); the level of ascorbate itself remained unaltered, but the levels of dehydroascorbate, threonate, and gulonate were reduced. IPA predicted dysregulation of the antioxidant action of vitamin $\mathrm{C}$ pathway in leiomyomas of the MED12 subtype based on both metabolic and gene expression data (Table 2). Similarly, retinol (vitamin A) was significantly reduced in the MED12 and triple wild-type subtypes (FC -2.5 and -1.8 , respectively). Based on previous studies, vitamin D deficiency contributes to the development of leiomyomas (Brakta et al, 2015); no vitamin D metabolites were detected in our study samples.

The alterations affecting amino-acid metabolites varied between leiomyoma subtypes. $\mathrm{FH}$-deficient leiomyomas displayed significant accumulation of urea cycle metabolites argininosuccinate (FC 38.5, Figure 3), proline (FC 1.5, Supplementary Figure S3), citrulline (FC 1.4), dimethylarginine (FC 2.5), trans-4-hydroxyproline (FC 1.7), and N-monomethylarginine (FC 1.6). The levels of leucine, isoleucine, and valine metabolites 3-methyl-2-oxobutyrate (FC 8.3), 4-methyl-2-oxopentanoate (FC 6.5), and 3-methyl-2- 

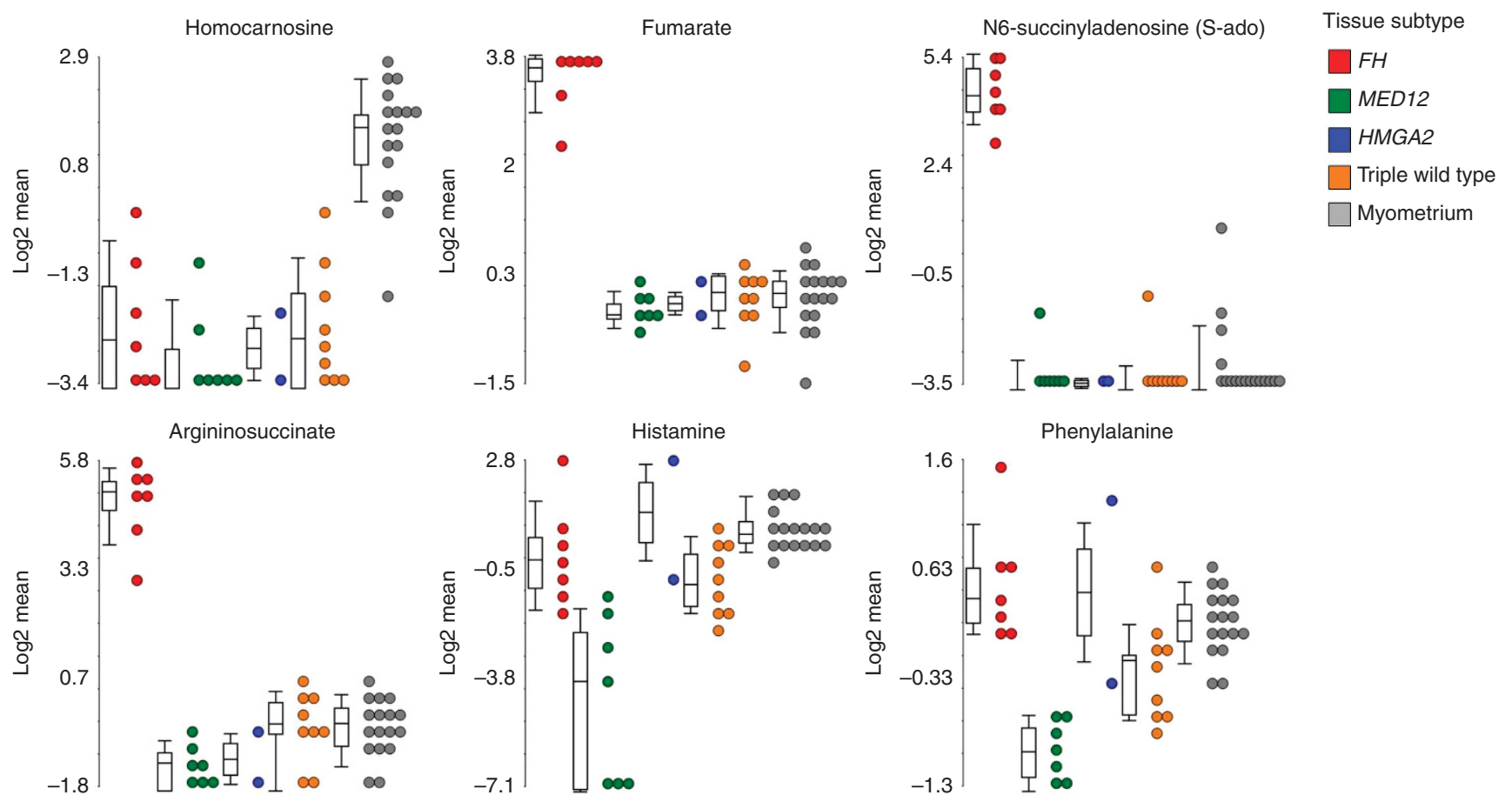

Figure 3. Levels of the most significantly dysregulated metabolites. Homocarnosine was the most significantly dysregulated metabolite in all leiomyomas. Fumarate, N6-succinyladenosine (S-ado), and argininosuccinate were the most uniquely dysregulated metabolites in leiomyomas of the FH subtype. Histamine and phenylalanine were the most uniquely dysregulated metabolites in leiomyomas of the MED12 subtype.

oxovalerate (FC 4.7) were notably elevated in leiomyomas of the $\mathrm{FH}$ subtype. Leiomyomas of the HMGA2 subtype displayed accretion of the polyamine metabolites $\mathrm{N}$-acetylputrescine (FC 11.3 ) and $\mathrm{N}(4)$-acetylspermidine (FC 7.7), the latter of which was also elevated in the FH subtype (FC 5.2). Furthermore, IPA predicted dysregulation of putrescine degradation III in the HMGA2 subtype based on both metabolomic and gene expression data (Table 2). In leiomyomas of the MED12 subtype, the pathway enrichment analysis of the metabolomic data revealed significant dysregulation of methionine, cysteine, S-adenosylmethionine (SAM), and taurine metabolism as well as significant reduction of dipeptides (Table 1). In addition, multiple amino acids and their derivatives were specifically decreased in the MED12 subtype, including histamine (FC - 35.9, Figure 3), phenylalanine (FC -2.2 , Figure 3 ), leucine (FC -1.5 , Supplementary Figure S3), isoleucine $(\mathrm{FC}-1.5)$, lysine $(\mathrm{FC}-1.5)$, arginine $(\mathrm{FC}-1.4)$, argininosuccinate $(\mathrm{FC}-1.9$, Figure 3$)$, tyrosine $(\mathrm{FC}-2.0)$, and tryptophan (FC -1.5$)$.

Leiomyomas displayed alterations in a wide array of lipid classes. The pathway enrichment analysis revealed significant dysregulation of sphingolipid metabolism in all subtypes except triple wild-type leiomyomas (Table 1). Especially leiomyomas of the MED12 subtype showed considerable enrichment of sphingolipid alterations characterised by significant reduction of 23 out of $38(60.5 \%)$ detected sphingolipids. In addition, IPA predicted dysregulation of sphingosine 1-phosphate signalling in the HMGA2 subtype at both the metabolic and transcriptional levels (Table 2). Phosphatidylserines were reduced especially in leiomyomas of the MED12 subtype (Table 1). Plasmalogens, diacylglycerols, and monohydroxy fatty acids were primarily elevated in both the FH and HMGA2 subtypes (Table 1, Supplementary Table S3).

As expected, several components of the TCA cycle were significantly elevated in leiomyomas of the $\mathrm{FH}$ subtype, including fumarate (FC 12.0, Figure 3), malate (FC 10.5, Supplementary Figure S3), alpha-ketoglutarate ( $\alpha \mathrm{KG}, \mathrm{FC} 5.1$ ), succinate (FC 4.1), and 2-methylcitrate/homocitrate (FC 2.0). The pathway enrichment analysis confirmed significant dysregulation of TCA cycle metabolism (Table 1). Both metabolomic and gene expression data suggested an increased flux through the pentose phosphate pathway (PPP): activation was supported by upregulation of PPP enzymes, such as glucose-6-phosphate dehydrogenase (FC 3.7), phosphogluconate dehydrogenase (FC 3.0), and transketolase (FC 4.3), and depletion of key metabolites (Tables 1 and 2).

To identify the most uniquely dysregulated metabolites as potential biomarkers for leiomyomas of the $F H$ and MED12 subtypes, we compared leiomyomas of these subtypes against all the other tumour and normal samples (Supplementary Table S4). We detected 240 and 75 subtype-specific metabolites ( $q$-value $<0.1$ ) in leiomyomas of the $F H$ and MED12 subtypes, respectively. Figure 3 illustrates the levels of the most uniquely dysregulated metabolites for both subtypes. To identify dysregulated main categories of metabolites, we performed metabolite set ANOVA. The FH subtype displayed a significant overall increase of energy, lipid, and nucleotide metabolites, whereas the MED12 subtype displayed a significant overall decrease of cofactors and vitamins, peptides, and unnamed metabolites, and the triple wild-type leiomyomas displayed significant reduction of cofactors and vitamins (Supplementary Figure S4).

\section{DISCUSSION}

This study explored the global metabolomic profiles of 25 uterine leiomyomas representing the three main molecular subtypes: leiomyomas driven by $F H$ deficiency, a MED12 mutation, or HMGA2 upregulation. Altogether 70 metabolites were commonly dysregulated in all leiomyomas. We found that $F H$-deficient leiomyomas represent, as expected, a metabolically distinct subtype of leiomyomas and that these leiomyomas can be distinguished based on their metabolomic profile, whereas the metabolic changes in the other subtypes were subtler. Leiomyomas of the $F H$ subtype displayed predominantly accumulation of metabolites, whereas the changes in the MED12 subtype were mainly decreases. It is intriguing to speculate that the overall reduction of metabolites 
Table 1. Significantly dysregulated pathways predicted from metabolomic data by pathway enrichment analysis for each leiomyoma subtype

\begin{tabular}{|c|c|c|c|c|}
\hline Pathway & Enrichment score & Enrichment $P$-value & $\begin{array}{c}\text { Proportion of significant } \\
\text { metabolites in pathway } \\
\text { (\%) }\end{array}$ & $\begin{array}{l}\text { Metabolites significantly } \\
\text { increased/decreased }\end{array}$ \\
\hline \multicolumn{5}{|l|}{$\mathrm{FH}$} \\
\hline Glycogen metabolism & 5.3 & 0.0048 & 4/4 (100.0\%) & $4 / 0$ \\
\hline TCA cycle & 5.1 & 0.0059 & $5 / 6(83.3 \%)$ & $5 / 0$ \\
\hline Sphingolipid metabolism & 4.6 & 0.0096 & 17/38 (44.7\%) & $7 / 10$ \\
\hline \multicolumn{5}{|l|}{ MED12 } \\
\hline Sphingolipid metabolism & 22.6 & $1.5 \times 10^{-10}$ & $23 / 38(60.5 \%)$ & $0 / 23$ \\
\hline Phosphatidylserine & 5.5 & 0.0042 & $3 / 3(100.0 \%)$ & $0 / 3$ \\
\hline $\begin{array}{l}\text { Ascorbate (vitamin C) and aldarate } \\
\text { metabolism }\end{array}$ & 4.2 & 0.015 & $3 / 4(75.0 \%)$ & $0 / 3$ \\
\hline Dipeptide & 3.2 & 0.043 & $4 / 9$ (44.4\%) & $0 / 4$ \\
\hline \multicolumn{5}{|l|}{ HMGA2 } \\
\hline Sphingolipid metabolism & 7.6 & 0.00053 & $8 / 38$ (21.1\%) & $1 / 7$ \\
\hline Diacylglycerol & 4.8 & 0.0085 & $4 / 16(25.0 \%)$ & $4 / 0$ \\
\hline Fatty acid, monohydroxy & 3.6 & 0.026 & $2 / 5(40.0 \%)$ & $2 / 0$ \\
\hline \multicolumn{5}{|l|}{ Triple wild type } \\
\hline Haemoglobin and porphyrin metabolism & 5.0 & 0.0065 & $2 / 4(50.0 \%)$ & $0 / 2$ \\
\hline $\begin{array}{l}\text { Ascorbate (vitamin C) and aldarate } \\
\text { metabolism }\end{array}$ & 5.0 & 0.0065 & $2 / 4(50.0 \%)$ & $0 / 2$ \\
\hline Dipeptide derivative & 3.4 & 0.034 & 1/1 (100.0\%) & $0 / 1$ \\
\hline Vitamin A metabolism & 3.4 & 0.034 & 1/1 (100.0\%) & $0 / 1$ \\
\hline
\end{tabular}

may limit the supply of building blocks and thereby explain the significantly smaller size of leiomyomas of the MED12 subtype (Heinonen et al, 2017).

Leiomyomas of all subtypes displayed reduction of specific vitamin and other cofactor metabolites, suggesting alterations in redox state, enzyme function, and signalling pathways. We detected a significant reduction of retinoic acid (RA) precursor retinol (vitamin A) in leiomyomas of the MED12 and triple wildtype subtypes. Furthermore, gene expression data revealed RA receptor activation as one of the most significantly dysregulated pathways in leiomyomas (Mehine et al, 2016). Reduced retinol levels may reflect a decreased concentration of biologically active RA and increased rate of RA metabolism, which has been previously observed in leiomyomas (Catherino and Malik, 2007). Furthermore, all-trans-RA, synthetic retinoid N-(4-hydroxyphenyl)retinamide (4-HPR), and RA metabolism-blocking agent liarozole have been shown to reduce leiomyoma cell proliferation and extracellular matrix production (Broaddus et al, 2004; Malik et al, 2008; Gilden et al, 2012). Essentially, 4-HPR and liarozole have been well tolerated in clinical studies (Debruyne et al, 1998; Camerini et al, 2001). The metabolites of ascorbate (vitamin C) were also depleted, and based on the gene expression data, the antioxidant action of vitamin $\mathrm{C}$ was predicted to be activated in leiomyomas of the MED12 subtype. In addition to antioxidant response, these alterations in vitamin $\mathrm{C}$ metabolites may reflect adaptations in other important cellular processes including DNA and histone demethylation, and the nuclear factor$\kappa \mathrm{B}$-mediated signalling (Cárcamo et al, 2004; Young et al, 2015). In particular, the depletion of vitamin $\mathrm{C}$ metabolites may link to the abundance of extracellular matrix in leiomyomas, as vitamin $\mathrm{C}$ is a cofactor in collagen synthesis (Du et al, 2012). Epidemiological studies have found no association between vitamin $\mathrm{C}$ and leiomyoma risk, whereas results regarding retinol levels and leiomyoma risk are contradictory (Martin et al, 2011; Wise et al, 2011), thus further research on their use as preventive agents is warranted.

As a novel finding, the levels of haeme, a fundamental co-factor in cellular respiration and signal transduction, and its degradation product biliverdin were reduced in all leiomyomas, suggesting that haeme metabolism has an important role in leiomyomagenesis. As the cataplerotic haeme biosynthesis uses TCA-cycle-derived carbon and haeme degradation produces bilirubin to be excreted, increased haeme metabolism promotes removal of accumulated TCA cycle metabolites especially in $\mathrm{FH}$-deficient leiomyomas, in which the expression of haeme-degrading-enzyme gene, haeme oxygenase 1 (HMOX1) is upregulated (Frezza et al, 2011; Mehine et al, 2016). Moreover, the inhibition of haeme biosynthesis and degradation pathway has demonstrated to be synthetic lethal with FH deficiency (Frezza et al, 2011). Although the mechanisms underlying these alterations in haeme metabolism are likely to be different in leiomyomas of the $F H$ subtype than in the other leiomyomas, targeting haeme metabolism may serve as valuable strategy to treat all leiomyomas, regardless of subtype.

The leiomyoma subtypes harboured multiple different alterations affecting peptide and amino-acid metabolites, which are necessary building blocks and energy sources for tumours. Homocarnosine, which is a dipeptide derivative predominantly found in the central nervous system, was abundant across all myometrium samples and detected at markedly reduced levels in all leiomyomas. The biological function of homocarnosine remains largely unclear, although as an antioxidant it may be important in counteracting oxidative stress (Kohen et al, 1988). No previous data seem to exist on the levels of homocarnosine in normal myometrium and further studies are required to understand the role of homocarnosine in this tissue type. Specifically, leiomyomas of the MED12 subtype displayed reduction of various amino acids, 
Table 2. Significantly dysregulated pathways at both the metabolome and transcriptome levels for leiomyomas of the FH, MED12, and HMGA2 subtypes ( $P$-value $<0.05$ in both metabolomic and gene expression data)

\begin{tabular}{|c|c|c|c|c|c|}
\hline & \multicolumn{3}{|r|}{ Gene expression data } & \multicolumn{2}{|r|}{ Metabolomic data } \\
\hline $\begin{array}{l}\text { Ingenuity canonical } \\
\text { pathways }\end{array}$ & $P$-value & Z-score & Genes & $P$-value & Metabolites \\
\hline \multicolumn{6}{|l|}{$\mathrm{FH}$} \\
\hline Pentose phosphate pathway & 0.041 & $\mathrm{NaN}$ & $P G D \uparrow, T K T \uparrow, G 6 P D \uparrow$ & 0.00004 & $\begin{array}{l}\text { Gluconic acid-6-phosphate } \downarrow \text {, } \\
\text { sedoheptulose 7-phosphate } \downarrow \text {, } \\
\text { D-ribulose 5-phosphate } \downarrow\end{array}$ \\
\hline $\begin{array}{l}\text { Pentose phosphate pathway } \\
\text { (oxidative branch) }\end{array}$ & 0.047 & $\mathrm{NaN}$ & $P G D \uparrow, G 6 P D \uparrow$ & 0.00041 & $\begin{array}{c}\text { Gluconic acid-6-phosphate } \downarrow \text {, } \\
\text { D-ribulose 5-phosphate } \downarrow\end{array}$ \\
\hline tRNA splicing & 0.0011 & $\mathrm{NaN}$ & $\begin{array}{c}P D E 10 A \downarrow, P D E 3 A \downarrow, P D E 1 B \uparrow, P D E 5 A \downarrow, P D E 8 B \uparrow, \\
P D E 1 A \uparrow, P D E 1 C \uparrow\end{array}$ & 0.0087 & Niacinamide $\uparrow$, NAD $+\downarrow$ \\
\hline Gluconeogenesis I & 0.025 & $\mathrm{NaN}$ & $P G K 1 \uparrow, E N O 1 \uparrow, E N O 2 \uparrow, M E 1 \uparrow, B P G M \uparrow$ & 0.0091 & L-malic acid $\uparrow, N A D+\downarrow$ \\
\hline IL-10 signalling & 0.042 & $\mathrm{NaN}$ & $\begin{array}{c}\text { IL33 } \uparrow, \text { HMOX } 1 \uparrow, \text { SOCS } \downarrow \downarrow \text {, FOS } \downarrow, J U N \downarrow \text {, } \\
\text { MAP4K } 4 \uparrow\end{array}$ & 0.020 & Haeme $\downarrow$, biliverdine $\downarrow$ \\
\hline $\begin{array}{l}\text { Salvage pathways of } \\
\text { pyrimidine ribonucleotides }\end{array}$ & 0.045 & $\mathrm{NaN}$ & $\begin{array}{c}\text { DAPK1 } \downarrow, A K 1 \uparrow, S G K 1 \downarrow, P R K A A 2 \downarrow, C D K 6 \uparrow, A K 4 \\
\uparrow, M A P 3 K 8 \downarrow, L I M K 1 \uparrow\end{array}$ & 0.046 & Uridine $\uparrow$, uracil $\uparrow$ \\
\hline \multicolumn{6}{|l|}{ MED12 } \\
\hline $\begin{array}{l}\text { Antioxidant action of } \\
\text { vitamin C }\end{array}$ & 0.0022 & 2.83 & $\begin{array}{c}\text { PLA2G4A } \downarrow, \text { PLCB4 } \downarrow, \text { PLCE1 } \downarrow, \text { NFKBIA } \downarrow, \text { PLCG2 } \\
\downarrow, \text { PLA2R1 } \downarrow, \text { MAPK10 } \downarrow, \text { RARRES3 } \downarrow, \text { SLC2A3 } \downarrow \text {, } \\
\text { TXNRD } 1 \downarrow\end{array}$ & 0.019 & $\begin{array}{l}\text { L-dehydroascorbic acid } \downarrow \text {, } \\
\text { glutathione disulphide } \downarrow\end{array}$ \\
\hline $\begin{array}{l}\text { Type II diabetes mellitus } \\
\text { signalling }\end{array}$ & 0.0025 & -1.00 & $\begin{array}{c}\text { PPARG } \downarrow, \text { SOCS3 } \downarrow, \text { NFKBIA } \downarrow, I R S 1 \uparrow, C D 36 \downarrow, \\
\text { ACSL5 } \downarrow, P R K A A 2 \downarrow, M A P K 10 \downarrow, I R S 4 \uparrow, A C S L 4 \downarrow, \\
\text { MAP3K5 } \downarrow, \text { PRKD } 1 \uparrow\end{array}$ & 0.034 & Sphingomyelin $\downarrow$, ceramide $\uparrow$ \\
\hline \multicolumn{6}{|l|}{ HMGA2 } \\
\hline $\begin{array}{l}\text { Putrescine degradation III } \\
\text { Sphingosine-1-phosphate } \\
\text { signalling }\end{array}$ & $\begin{array}{l}0.028 \\
0.0035\end{array}$ & $\begin{array}{l}\mathrm{NaN} \\
-1.51\end{array}$ & $\begin{array}{c}\text { ALDH1B1 } \downarrow, \text { MAOB } \downarrow, \text { ALDH1A1 } \downarrow, \text { MAOA } \downarrow \\
\text { S1PR3 } \uparrow, P L C B 4 \downarrow, P L C E 1 \downarrow, R N D 3 \downarrow, P L C G 2 \downarrow, \\
\text { PDGFRA } \downarrow, \text { GNAI1 } \downarrow \text {, PIK3C2G } \uparrow, \text { AKT3 } \downarrow \text {, PDGFD } \\
\downarrow, \text { PDGFC } \uparrow\end{array}$ & $\begin{array}{l}0.011 \\
0.039\end{array}$ & $\begin{array}{l}\text { N-acetylputrescine } \uparrow \\
\text { Sphingomyelin } \downarrow\end{array}$ \\
\hline
\end{tabular}

their derivatives, and dipeptides, possibly reflecting increased protein synthesis. These leiomyomas showed also enrichment of dysregulated methionine, cysteine, SAM, and taurine metabolites. The observed reduction of taurine is compatible with a previous report (Mazzei et al, 2010). As SAM is the major coenzyme in reactions with methyl group transfer, these changes may reflect altered methylation of proteins and DNA in leiomyomas of the MED12 subtype (Teperino et al, 2010; Pavlova and Thompson, 2016). On the contrary, FH-deficient leiomyomas displayed accretion of valine, leucine, and isoleucine metabolites, suggesting that blockage of the TCA cycle impairs the function of the branched-chain alpha-keto acid dehydrogenase complex, which belongs to the mitochondrial dehydrogenase complex family and is essential in the metabolism of these amino acids (Harper et al, 1984). In addition, leiomyomas of the $F H$ and $H M G A 2$ subtypes displayed accretion of polyamines, which has been implicated in induction of cell proliferation and reduction of apoptosis (Gerner and Meyskens, 2004). Targeting polyamine metabolism may prove an effective therapy or, more importantly, a prevention mechanism for these leiomyoma subtypes, as indicated by a prior study showing that treatment with 2-difluoromethylornithine, a welltolerated inhibitor of a central enzyme in polyamine synthesis, limits the growth of leiomyoma cells (Broaddus et al, 2004; Gerner and Meyskens, 2004).

All leiomyomas displayed alterations in a wide array of lipid classes, yet with subtype specific differences. Levels of sphingolipids and phosphatidylserines, which are structural components of cell membranes, were especially reduced in leiomyomas of the MED12 subtype. Sphingolipids are signal transducers that regulate apoptosis and proliferation (Ogretmen and Hannun, 2004). Phosphatidylserines also have a role in signalling pathways and they mediate humoral and cellular processes, including blood coagulation and phagocytosis (Vance and Steenbergen, 2005). In contrast, other important components of cell membranes, plasmalogens and diacylglycerols, were increased in leiomyomas of the FH and HMGA2 subtypes. These lipids affect the membrane properties and facilitate various signalling processes (GómezFernández and Corbalán-García, 2007; Braverman and Moser, 2012). All these deregulations in lipid metabolites presumably contribute to leiomyoma development on many levels, not only by providing an energy source and building blocks for membrane biogenesis during cell growth but also by regulating signalling pathways involved in proliferation and differentiation.

The most apparent effect of $F H$ deficiency was intracellular fumarate accumulation, which is considered tumour promoting (Adam et al, 2011). Additionally, FH-deficient leiomyomas displayed accumulation of other TCA cycle intermediates malate, succinate, $\alpha \mathrm{KG}$, and homocitrate. Prior studies using HLRCCrelated tumours, $\mathrm{FH}$-deficient kidney cells and tissues from mice, and a metastatic kidney cancer cell line from an HLRCC patient have detected accumulation of fumarate and succinate but have provided contradicting data on the levels of malate, $\alpha \mathrm{KG}$, and citrate (Pollard et al, 2005; Frezza et al, 2011; Tong et al, 2011; Adam et al, 2013; Yang et al, 2013; Zheng et al, 2013). Furthermore, patients with congenital fumarase deficiency, a rare autosomal-recessive disorder, display increased levels of fumarate, succinate, $\alpha \mathrm{KG}$, citrate, and malate in their physiological fluids (Allegri et al, 2010; Tregoning et al, 2013). Mullen et al (2012) demonstrated that human-derived $\mathrm{FH}$-deficient kidney cancer cells use glutamine-dependent reductive carboxylation of $\alpha \mathrm{KG}$ to produce citrate, acetyl coenzyme $\mathrm{A}$, and remaining four-carbon TCA intermediates. This reductive carboxylation supports fatty acid synthesis allowing $\mathrm{FH}$-deficient cells to maintain growth (Mullen et al, 2012). Studies using FH-deficient mouse cell lines, however, observed no evidence of reductive carboxylation (Frezza et al, 2011; Adam et al, 2013). As increased $\alpha \mathrm{KG} /$ citrate ratio 
triggers reductive glutamine metabolism (Fendt et al, 2013), our results suggest that reductive carboxylation occurs in $\mathrm{FH}$-deficient leiomyomas, but isotope-labelling studies using leiomyoma cell lines would be required for confirmation.

Based on the current knowledge of the tumourigenic mechanism of FH-deficiency, accumulation of intracellular fumarate activates transcription factor nuclear factor (erythroid-derived 2)like 2 (NRF2) (Adam et al, 2011). NRF2 is a master regulator of antioxidant responses and it redirects glucose and glutamine into anabolic pathways including the PPP, which is an efficient source of NADPH (Mitsuishi et al, 2012; Gorrini et al, 2013). The gene expression data revealed a highly significant activation of NRF2mediated oxidative stress response in leiomyomas of the $\mathrm{FH}$ subtype causing, for example, upregulation of HMOX1 (Gorrini et al, 2013; Mehine et al, 2016). This is further supported by our metabolomic and gene expression data as $\mathrm{FH}$-deficient leiomyomas displayed several metabolic alterations known to be affected by activated NRF2 including increased PPP flux, validating an observation made previously in FH-deficient kidney cancer cell lines (Yang et al, 2013). All in all, NRF2 activation supports FHdeficient cell proliferation by enhancing PPP and generating $\mathrm{NADPH}$, which is critical for reductive carboxylation of glutaminederived $\alpha \mathrm{KG}$, synthesis of fatty acids, and protection against oxidative stress.

Our analysis discovered putative biomarkers for $\mathrm{FH}$ deficiency including argininosuccinate and N6-succinyladenosine (S-ado). Argininosuccinate is a precursor to fumarate via argininosuccinate lyase (ASL) and S-ado is formed by dephosporylation of adenylosuccinate, a substrate of adenylosuccinate lyase (ADSL) that normally catalyzes formation of fumarate. $\mathrm{FH}$-deficient cells seem to control the levels of fumarate by reversing or inhibiting the reactions catalyzed by ASL and ADSL leading to argininosuccinate and S-ado accumulation (Adam et al, 2013; Zheng et al, 2013; Jurecka et al, 2015). FH-deficient kidney specimens from mice as well as physiological fluids of patients with congenital fumarase deficiency display increased levels of adenylosuccinate, providing further support for the inhibition of ADSL activity in FH-deficient tissues (Allegri et al, 2010; Adam et al, 2013; Tregoning et al, 2013). The usefulness of S-ado as a biomarker and its tumourigenic role require further research. Argininosuccinate is normally produced in the urea cycle. FH-deficient kidney cancers employ exogenous arginine to produce argininosuccinate and blocking this mechanism reduces proliferation of these cells, suggesting that the arginine pathway is essential for the growth of $\mathrm{FH}$-deficient tumours (Adam et al, 2013; Zheng et al, 2013). Intriguingly, clinical studies have revealed a tolerable profile for an arginine-depleting drug (Ott et al, 2013). Furthermore, Zheng et al (2013) observed abundant secretion of argininosuccinate into the urine of Fh1-deficient mice and into the growth media of $F H$-deficient cell lines. Thus, argininosuccinate and arginine metabolism may prove to be a robust metabolic biomarker and a treatment target for HLRCCassociated tumours, respectively.

Taken together, this study discovered novel metabolic alterations in leiomyomas involving vitamin $\mathrm{C}$, various membrane lipid, peptide, and amino-acid metabolites (Figure 4), which may be essential for the growth of specific leiomyoma subtypes. Our findings show the importance of haeme and polyamine metabolism and validate the dysregulation of vitamin $\mathrm{A}$ in primary leiomyoma tissues. The results revealed the consequences of impaired TCA cycle associated with $F H$-deficient leiomyomas pinpointing biomarkers and potential therapeutic targets. Importantly, welltolerated drugs tested in patient trials exist for many of these aberrant states.

To our knowledge, this is the first study that employs a global metabolomics approach to analyse uterine leiomyomas and corresponding myometrium. We have previously shown that the main genetic drivers of uterine leiomyomas result in distinct gene
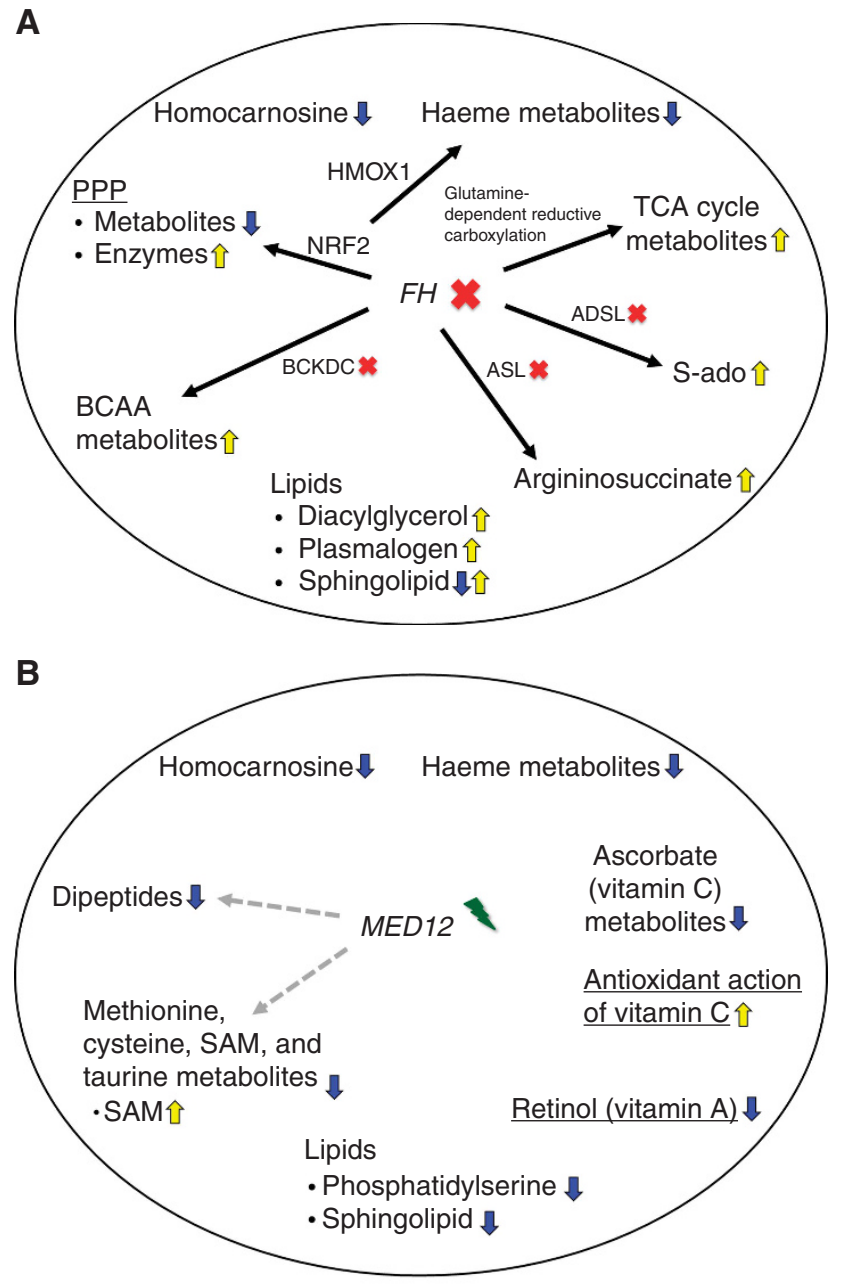
* Compromised enzyme function
- Oncogenic mutation
î Statistically significant increase
V Statistically significant decrease
$\rightarrow$ Putative mechanism based on metabolomic data and literature
$\rightarrow$ Suggestive association based on significantly dysregulated pathway predicted from metabolomic data unique to this subtype
Significantly dysregulated metabolites/pathways predicted from both metabolomic and gene expression data

Figure 4. Schematic of the highlighted metabolic alterations in leiomyomas of the FH and MED12 subtypes. All leiomyomas displayed reduced homocarnosine and haeme metabolite levels and subtypespecific alterations in lipids. (A) FH-deficient leiomyomas displayed dysregulation of TCA cycle, PPP, and the metabolism of amino acids and nucleotides. (B) MED12-mutated leiomyomas harboured alterations affecting retinol, ascorbate, amino acids, and dipeptides. $\mathrm{ADSL}=$ adenylosuccinate lyase; $\mathrm{ASL}=$ argininosuccinate lyase; $B C A A=$ branched chain amino acid; $B C K D C=$ branched-chain alphaketo acid dehydrogenase complex; $\mathrm{FH}=$ fumarate hydratase; HMOX1 = haeme oxygenase $1 ;$ MED12=mediator complex subunit 12; NRF2 = nuclear factor (erythroid-derived 2)-like 2; PPP = pentose phosphate pathway; S-ado = N6-succinyladenosine; SAM =Sadenosylmethionine; TCA $=$ tricaboxylic acid.

expression profiles (Mehine et al, 2016). Here we show that these genetic drivers lead to differences in the metabolomic profiles as well. Our results highlight the diversity of metabolic alterations that can underlie a similar clinical phenotype. The dysregulated metabolites identified in this study may prove to be useful biomarkers and targets for prevention and treatment of uterine leiomyomas, although future studies with larger sample sets are 
warranted, especially for leiomyomas with HMGA2 overexpression. Furthermore, metabolic flux studies are required to determine whether the observed changes are derived from altered flux through a pathway or altered synthesis, degradation, or transport of the metabolites. The metabolomic profile of uterine leiomyomas will contribute to understanding pathophysiology of uterine leiomyomas and provides a promising foundation for therapeutic development.

\section{ACKNOWLEDGEMENTS}

We sincerely thank $\mathrm{P}$ Ikonen, S Nieminen, M Rajalaakso, I-L Svedberg, I Vuoristo, and the staffs of the Department of Pathology and Department of Obstetrics and Gynecology, Helsinki University Hospital for technical assistance; and Metabolomics Unit, Technology Centre, Institute for Molecular Medicine Finland FIMM, University of Helsinki; the Biomedicum Functional Genomics Unit; the Estonian Genome Center, University of Tartu; and Metabolon Inc. for their services. This study was supported by the Academy of Finland (Center of Excellence in Cancer Genetics Research 20122017, 250345), European Research Council (ERC, 695727), as well as the Cancer Society of Finland, the Maud Kuistila Foundation, the Ida Montin Foundation, the Biomedicum Helsinki Foundation, Kliinisen Kemian Tutkimussäätiö, K. Albin Johansson Foundation, Orion Research Foundation sr, and the Finnish-Norwegian Medical Foundation (personal grants to $\mathrm{H}-\mathrm{RH}$ ).

\section{CONFLICT OF INTEREST}

The authors declare no conflict of interest.

\section{REFERENCES}

Adam J, Hatipoglu E, O'Flaherty L, Ternette N, Sahgal N, Lockstone H, Baban D, Nye E, Stamp GW, Wolhuter K, Stevens M, Fischer R, Carmeliet P, Maxwell PH, Pugh CW, Frizzell N, Soga T, Kessler BM, El-Bahrawy M, Ratcliffe PJ, Pollard PJ (2011) Renal cyst formation in Fh1-deficient mice is independent of the Hif/Phd pathway: roles for fumarate in KEAP1 succination and Nrf2 signaling. Cancer Cell 20: 524-537.

Adam J, Yang M, Bauerschmidt C, Kitagawa M, O’Flaherty L, Maheswaran P, Özkan G, Sahgal N, Baban D, Kato K, Saito K, Iino K, Igarashi K, Stratford M, Pugh C, Tennant DA, Ludwig C, Davies B, Ratcliffe PJ, El-Bahrawy M, Ashrafian H, Soga T, Pollard PJ (2013) A role for cytosolic fumarate hydratase in urea cycle metabolism and renal neoplasia. Cell Rep 3: $1440-1448$

Allegri G, Fernandes MJ, Scalco FB, Correia P, Simoni RE, Llerena JC Jr, de Oliveira ML (2010) Fumaric aciduria: an overview and the first Brazilian case report. J Inherit Metab Dis 33: 411-419.

Baird DD, Dunson DB, Hill MC, Cousins D, Schectman JM (2003) High cumulative incidence of uterine leiomyoma in black and white women: ultrasound evidence. Am J Obstet Gynecol 188: 100-107.

Benjamini Y, Hochberg Y (1995) Controlling the false discovery rate: a practical and powerful approach to multiple testing. J R Stat Soc Series B Stat Methodol 57: 289-300.

Brakta S, Diamond JS, Al-Hendy A, Diamond MP, Halder SK (2015) Role of vitamin D in uterine fibroid biology. Fertil Steril 104: 698-706.

Braverman NE, Moser AB (2012) Functions of plasmalogen lipids in health and disease. Biochim Biophys Acta 1822: 1442-1452.

Broaddus RR, Xie S, Hsu CJ, Wang J, Zhang S, Zou C (2004) The chemopreventive agents 4-HPR and DFMO inhibit growth and induce apoptosis in uterine leiomyomas. Am J Obstet Gynecol 190: 686-692.

Camerini T, Mariani L, De Palo G, Marubini E, Di Mauro MG, Decensi A, Costa A, Veronesi U (2001) Safety of the synthetic retinoid fenretinide: long-term results from a controlled clinical trial for the prevention of contralateral breast cancer. J Clin Oncol 19: 1664-1670.
Cardozo ER, Clark AD, Banks NK, Henne MB, Stegmann BJ, Segars JH (2012) The estimated annual cost of uterine leiomyomata in the United States. Am J Obstet Gynecol 206: 211.e1-211.e9.

Catherino WH, Malik M (2007) Uterine leiomyomas express a molecular pattern that lowers retinoic acid exposure. Fertil Steril 87: 1388-1398.

Cramer SF, Patel A (1990) The frequency of uterine leiomyomas. Am J Clin Pathol 94: 435-438.

Cárcamo JM, Pedraza A, Bórquez-Ojeda O, Zhang B, Sanchez R, Golde DW (2004) Vitamin C is a kinase inhibitor: dehydroascorbic acid inhibits IkappaBalpha kinase beta. Mol Cell Biol 24: 6645-6652.

Debruyne FJ, Murray R, Fradet Y, Johansson JE, Tyrrell C, Boccardo F, Denis L, Marberger JM, Brune D, Rassweiler J, Vangeneugden T, Bruynseels J, Janssens M, De Porre P (1998) Liarozole-a novel treatment approach for advanced prostate cancer: results of a large randomized trial versus cyproterone acetate. Liarozole Study Group. Urology 52: 72-81.

Du J, Cullen JJ, Buettner GR (2012) Ascorbic acid: chemistry, biology and the treatment of cancer. Biochim Biophys Acta 1826: 443-457.

Fendt SM, Bell EL, Keibler MA, Olenchock BA, Mayers JR, Wasylenko TM, Vokes NI, Guarente L, Vander Heiden MG, Stephanopoulos G (2013) Reductive glutamine metabolism is a function of the alpha-ketoglutarate to citrate ratio in cells. Nat Commun 4: 2236.

Frezza C, Zheng L, Folger O, Rajagopalan KN, MacKenzie ED, Jerby L, Micaroni M, Chaneton B, Adam J, Hedley A, Kalna G, Tomlinson IP, Pollard PJ, Watson DG, Deberardinis RJ, Shlomi T, Ruppin E, Gottlieb E (2011) Haem oxygenase is synthetically lethal with the tumour suppressor fumarate hydratase. Nature 477: 225-228.

Gerner EW, Meyskens FL Jr (2004) Polyamines and cancer: old molecules, new understanding. Nat Rev Cancer 4: 781-792.

Gilden M, Malik M, Britten J, Delgado T, Levy G, Catherino WH (2012) Leiomyoma fibrosis inhibited by liarozole, a retinoic acid metabolic blocking agent. Fertil Steril 98: 1557-1562.

Gorrini C, Harris IS, Mak TW (2013) Modulation of oxidative stress as an anticancer strategy. Nat Rev Drug Discov 12: 931-947.

Gómez-Fernández JC, Corbalán-García S (2007) Diacylglycerols, multivalent membrane modulators. Chem Phys Lipids 148: 1-25.

Harper AE, Miller RH, Block KP (1984) Branched-chain amino acid metabolism. Annu Rev Nutr 4: 409-454.

Heinonen HR, Pasanen A, Heikinheimo O, Tanskanen T, Palin K, Tolvanen J, Vahteristo P, Sjöberg J, Pitkänen E, Bützow R, Mäkinen N, Aaltonen LA (2017) Multiple clinical characteristics separate MED12-mutation-positive and -negative uterine leiomyomas. Sci Rep 7: 1015.

Heinonen HR, Sarvilinna NS, Sjöberg J, Kämpjärvi K, Pitkänen E, Vahteristo P, Mäkinen N, Aaltonen LA (2014) MED12 mutation frequency in unselected sporadic uterine leiomyomas. Fertil Steril 102: $1137-1142$.

Jurecka A, Zikanova M, Kmoch S, Tylki-Szymańska A (2015) Adenylosuccinate lyase deficiency. J Inherit Metab Dis 38: 231-242.

Kohen R, Yamamoto Y, Cundy KC, Ames BN (1988) Antioxidant activity of carnosine, homocarnosine, and anserine present in muscle and brain. Proc Natl Acad Sci USA 85: 3175-3179.

Kämpjärvi K, Mäkinen N, Mehine M, Välipakka S, Uimari O, Pitkänen E, Heinonen H, Heikkinen T, Tolvanen J, Ahtikoski A, Frizzell N, Sarvilinna N, Sjöberg J, Bützow R, Aaltonen LA, Vahteristo P (2016) MED12 mutations and FH inactivation are mutually exclusive in uterine leiomyomas. Br J Cancer 114: 1405-1411.

Lehtonen R, Kiuru M, Vanharanta S, Sjöberg J, Aaltonen LM, Aittomäki K, Arola J, Bützow R, Eng C, Husgafvel-Pursiainen K, Isola J, Järvinen H, Koivisto P, Mecklin JP, Peltomäki P, Salovaara R, Wasenius VM, Karhu A, Launonen V, Nupponen NN, Aaltonen LA (2004) Biallelic inactivation of fumarate hydratase $(\mathrm{FH})$ occurs in nonsyndromic uterine leiomyomas but is rare in other tumors. Am J Pathol 164: 17-22.

Malik M, Norian J, McCarthy-Keith D, Britten J, Catherino WH (2010) Why leiomyomas are called fibroids: the central role of extracellular matrix in symptomatic women. Semin Reprod Med 28: 169-179.

Malik M, Webb J, Catherino WH (2008) Retinoic acid treatment of human leiomyoma cells transformed the cell phenotype to one strongly resembling myometrial cells. Clin Endocrinol (Oxf) 69: 462-470.

Markowski DN, Bartnitzke S, Löning T, Drieschner N, Helmke BM, Bullerdiek J (2012) MED12 mutations in uterine fibroids-their relationship to cytogenetic subgroups. Int J Cancer 131: 1528-1536.

Martin CL, Huber LR, Thompson ME, Racine EF (2011) Serum micronutrient concentrations and risk of uterine fibroids. J Womens Health (Larchmt) 20: 915-922. 
Mazzei P, Piccolo A, Nugnes L, Mascolo M, De Rosa G, Staibano S (2010) Metabolic profile of intact tissue from uterine leiomyomas using highresolution magic-angle-spinning ${ }^{1} \mathrm{H}$ NMR spectroscopy. NMR Biomed 23: $1137-1145$.

Mehine M, Kaasinen E, Heinonen HR, Mäkinen N, Kämpjärvi K, Sarvilinna $\mathrm{N}$, Aavikko M, Vähärautio A, Pasanen A, Bützow R, Heikinheimo O, Sjöberg J, Pitkänen E, Vahteristo P, Aaltonen LA (2016) Integrated data analysis reveals uterine leiomyoma subtypes with distinct driver pathways and biomarkers. Proc Natl Acad Sci USA 113: 1315-1320.

Mehine M, Mäkinen N, Heinonen HR, Aaltonen LA, Vahteristo P (2014) Genomics of uterine leiomyomas: insights from high-throughput sequencing. Fertil Steril 102: 621-629.

Mesnage R, Agapito-Tenfen SZ, Vilperte V, Renney G, Ward M, Séralini GE, Nodari RO, Antoniou MN (2016) An integrated multi-omics analysis of the NK603 Roundup-tolerant GM maize reveals metabolism disturbances caused by the transformation process. Sci Rep 6: 37855 .

Mitsuishi Y, Taguchi K, Kawatani Y, Shibata T, Nukiwa T, Aburatani H, Yamamoto M, Motohashi H (2012) Nrf2 redirects glucose and glutamine into anabolic pathways in metabolic reprogramming. Cancer Cell 22: 66-79.

Mullen AR, Wheaton WW, Jin ES, Chen P, Sullivan LB, Cheng T, Yang Y, Linehan WM, Chandel NS, DeBerardinis RJ (2012) Reductive carboxylation supports growth in tumour cells with defective mitochondria. Nature 481: 385-388.

Mäkinen N, Mehine M, Tolvanen J, Kaasinen E, Li Y, Lehtonen HJ, Gentile M, Yan J, Enge M, Taipale M, Aavikko M, Katainen R, Virolainen E, Böhling T, Koski TA, Launonen V, Sjöberg J, Taipale J, Vahteristo P, Aaltonen LA (2011) MED12, the mediator complex subunit 12 gene, is mutated at high frequency in uterine leiomyomas. Science 334: 252-255.

Nikkanen J, Forsström S, Euro L, Paetau I, Kohnz RA, Wang L, Chilov D, Viinamäki J, Roivainen A, Marjamäki P, Liljenbäck H, Ahola S, Buzkova J, Terzioglu M, Khan NA, Pirnes-Karhu S, Paetau A, Lönnqvist T, Sajantila A, Isohanni P, Tyynismaa H, Nomura DK, Battersby BJ, Velagapudi V, Carroll CJ, Suomalainen A (2016) Mitochondrial DNA replication defects disturb cellular dNTP pools and remodel one-carbon metabolism. Cell Metab 23: 635-648.

Ogretmen B, Hannun YA (2004) Biologically active sphingolipids in cancer pathogenesis and treatment. Nat Rev Cancer 4: 604-616.

Ott PA, Carvajal RD, Pandit-Taskar N, Jungbluth AA, Hoffman EW, Wu BW, Bomalaski JS, Venhaus R, Pan L, Old LJ, Pavlick AC, Wolchok JD (2013) Phase I/II study of pegylated arginine deiminase (ADI-PEG 20) in patients with advanced melanoma. Invest New Drugs 31: 425-434.

Pavlova NN, Thompson CB (2016) The emerging hallmarks of cancer metabolism. Cell Metab 23: 27-47.

Pollard PJ, Brière JJ, Alam NA, Barwell J, Barclay E, Wortham NC, Hunt T, Mitchell M, Olpin S, Moat SJ, Hargreaves IP, Heales SJ, Chung YL, Griffiths JR, Dalgleish A, McGrath JA, Gleeson MJ, Hodgson SV, Poulsom R, Rustin P, Tomlinson IP (2005) Accumulation of Krebs cycle intermediates and over-expression of HIF1a in tumours which result from germline FH and SDH mutations. Hum Mol Genet 14: 2231-2239.
Schoenmakers EF, Wanschura S, Mols R, Bullerdiek J, Van den Berghe H, Van de Ven WJ (1995) Recurrent rearrangements in the high mobility group protein gene, HMGI-C, in benign mesenchymal tumours. Nat Genet 10: 436-444.

Stovall DW (2001) Clinical symptomatology of uterine leiomyomas. Clin Obstet Gynecol 44: 364-371.

Tennant DA, Durán RV, Gottlieb E (2010) Targeting metabolic transformation for cancer therapy. Nat Rev Cancer 10: 267-277.

Teperino R, Schoonjans K, Auwerx J (2010) Histone methyl transferases and demethylases; can they link metabolism and transcription? Cell Metab 12 321-327.

Tomlinson IP, Alam NA, Rowan AJ, Barclay E, Jaeger EE, Kelsell D, Leigh I, Gorman P, Lamlum H, Rahman S, Roylance RR, Olpin S, Bevan S, Barker K, Hearle N, Houlston RS, Kiuru M, Lehtonen R, Karhu A, Vilkki S, Laiho P, Eklund C, Vierimaa O, Aittomäki K, Hietala M, Sistonen P, Paetau A, Salovaara R, Herva R, Launonen V, Aaltonen LA, Multiple Leiomyoma Consortium (2002) Germline mutations in FH predispose to dominantly inherited uterine fibroids, skin leiomyomata and papillary renal cell cancer. Nat Genet 30: 406-410.

Tong WH, Sourbier C, Kovtunovych G, Jeong SY, Vira M, Ghosh M, Romero VV, Sougrat R, Vaulont S, Viollet B, Kim YS, Lee S, Trepel J, Srinivasan R, Bratslavsky G, Yang Y, Linehan WM, Rouault TA (2011) The glycolytic shift in fumarate-hydratase-deficient kidney cancer lowers AMPK levels, increases anabolic propensities and lowers cellular iron levels. Cancer Cell 20: 315-327.

Tregoning S, Salter W, Thorburn DR, Durkie M, Panayi M, Wu JY, Easterbrook A, Coman DJ (2013) Fumarase deficiency in dichorionic diamniotic twins. Twin Res Hum Genet 16: 1117-1120.

Vance JE, Steenbergen R (2005) Metabolism and functions of phosphatidylserine. Prog Lipid Res 44: 207-234.

Wise LA, Radin RG, Palmer JR, Kumanyika SK, Boggs DA, Rosenberg L (2011) Intake of fruit, vegetables, and carotenoids in relation to risk of uterine leiomyomata. Am J Clin Nutr 94: 1620-1631.

Yang Y, Lane AN, Ricketts CJ, Sourbier C, Wei MH, Shuch B, Pike L, Wu M, Rouault TA, Boros LG, Fan TW, Linehan WM (2013) Metabolic reprogramming for producing energy and reducing power in fumarate hydratase null cells from hereditary leiomyomatosis renal cell carcinoma. PLoS One 8: e72179.

Young JI, Züchner S, Wang G (2015) Regulation of the epigenome by vitamin C. Annu Rev Nutr 35: 545-564.

Zheng L, MacKenzie ED, Karim SA, Hedley A, Blyth K, Kalna G, Watson DG, Szlosarek P, Frezza C, Gottlieb E (2013) Reversed argininosuccinate lyase activity in fumarate hydratase-deficient cancer cells. Cancer Metab 1: 12.

This work is published under the standard license to publish agreement. After 12 months the work will become freely available and the license terms will switch to a Creative Commons AttributionNonCommercial-Share Alike 4.0 Unported License.

Supplementary Information accompanies this paper on British Journal of Cancer website (http://www.nature.com/bjc) 